Supplement of Geosci. Model Dev., 13, 3975-3993, 2020

https://doi.org/10.5194/gmd-13-3975-2020-supplement

(C) Author(s) 2020. This work is distributed under

the Creative Commons Attribution 4.0 License.

(c) (1)

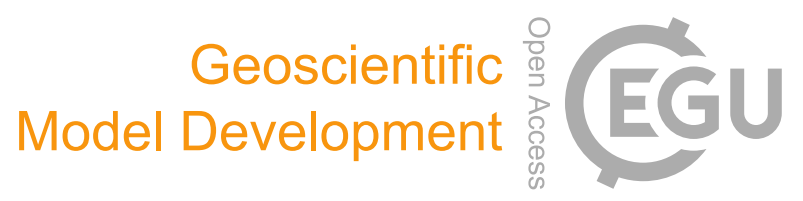

Supplement of

\title{
Role of vegetation in representing land surface temperature in the CHTESSEL (CY45R1) and SURFEX-ISBA (v8.1) land surface models: a case study over Iberia
}

Miguel Nogueira et al.

Correspondence to: Miguel Nogueira (mdnogueira@fc.ul.pt)

The copyright of individual parts of the supplement might differ from the CC BY 4.0 License. 

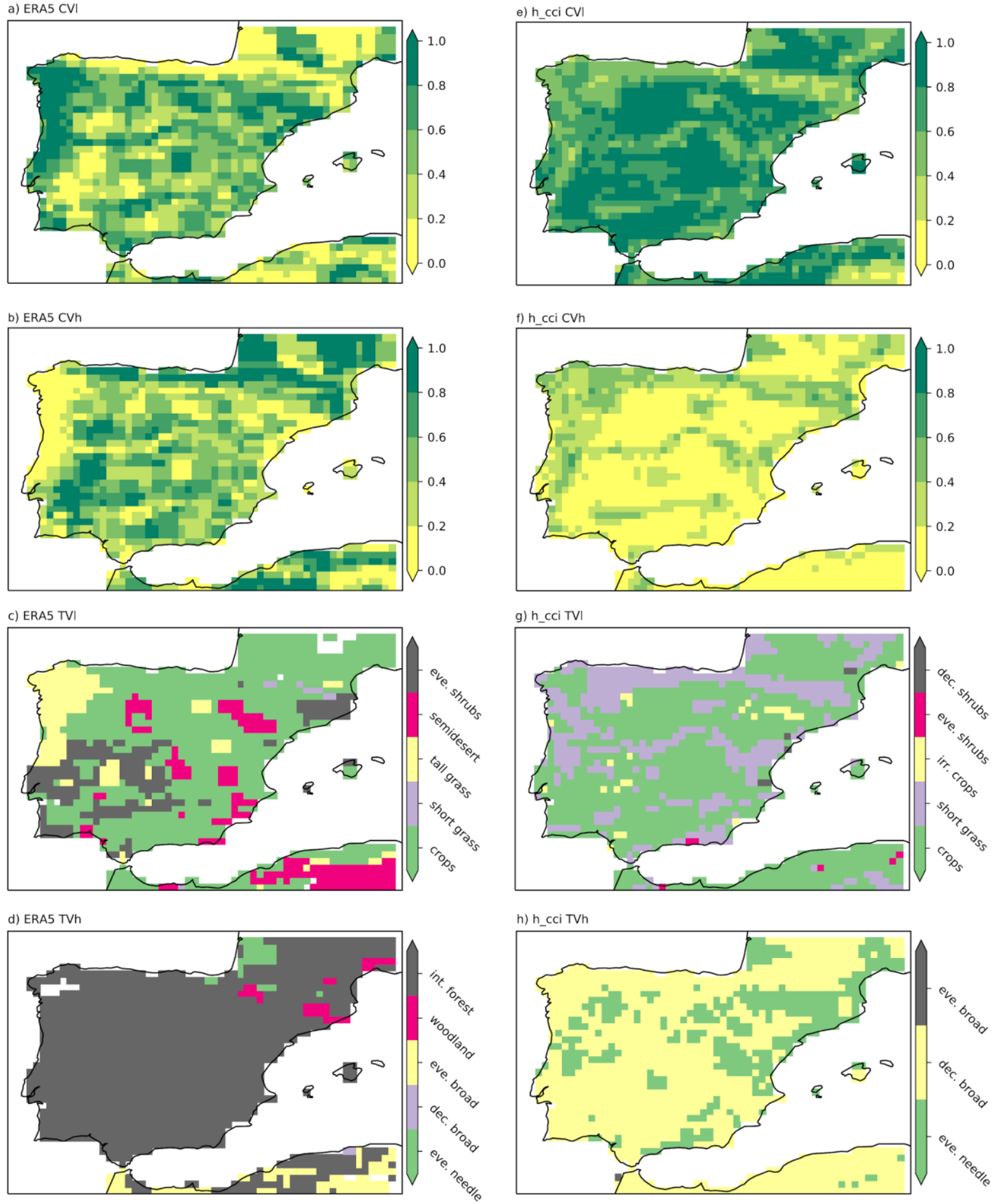

Supplementary Figure S1. Comparison of ERA5 (a-d and CTR) with H_CCI (e-h) vegetation cover and dominant vegetation types: CVl $(\mathrm{a}, \mathrm{e}), \mathrm{CVh}(\mathrm{b}, \mathrm{f}), \mathrm{TVl}(\mathrm{c}, \mathrm{g}), \mathrm{TVh}(\mathrm{d}, \mathrm{h})$. Note the different color and associated dominant vegetation types in panels $\mathrm{c}, \mathrm{g}, \mathrm{d}, \mathrm{h}$. 


\section{a) ERA5}

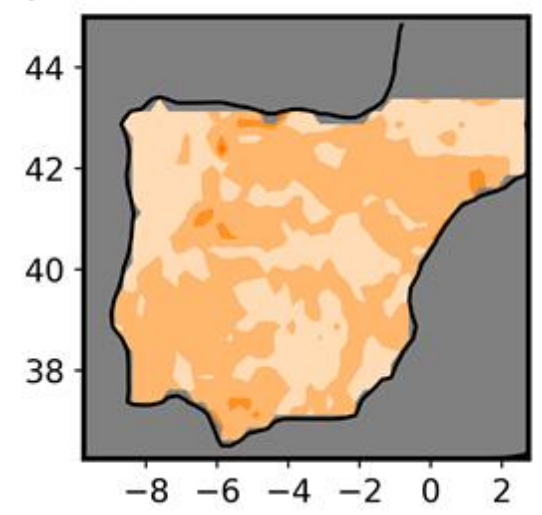

c) SFX

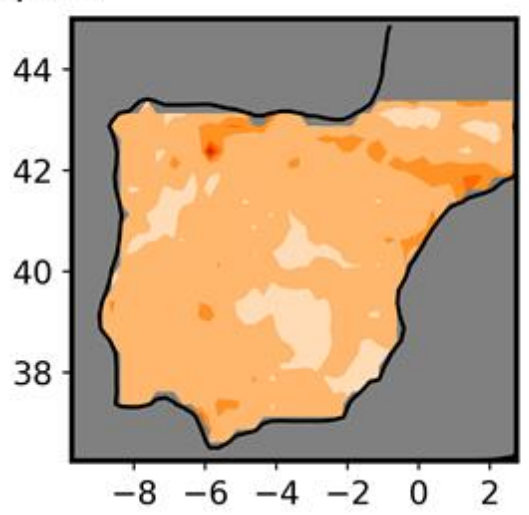

e) H_CCl_cl

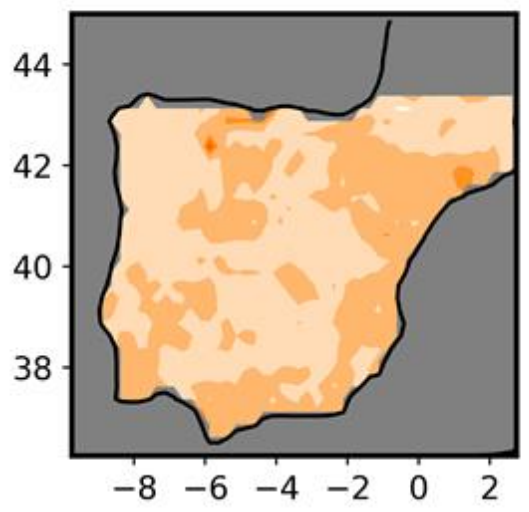

b) CTR

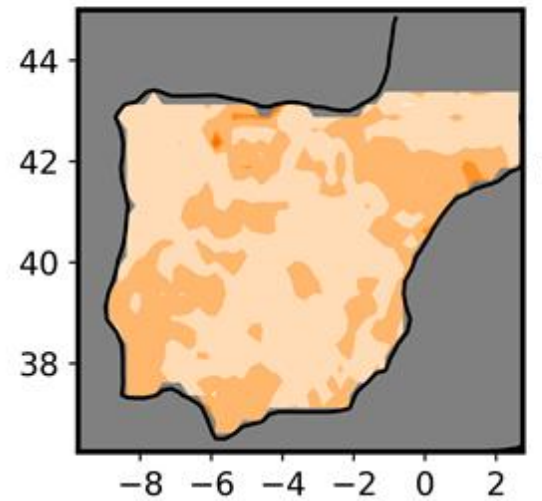

d) $\mathrm{H} \_\mathrm{CCl}$

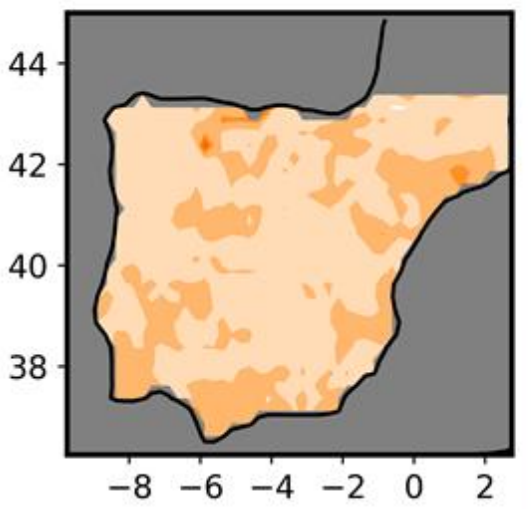

f) H_CCl_cl_LAI

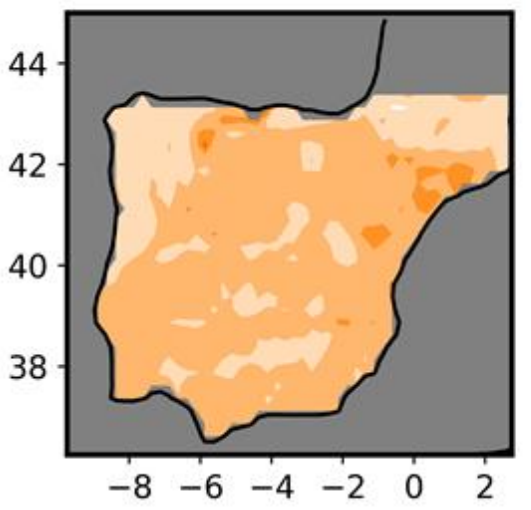

UbRMSE

[ $\left.{ }^{\circ} \mathrm{C}\right]$

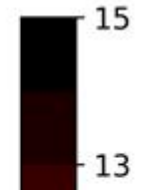

11

9

7

5

Supplementary Figure S2. Maps of JJA daily maximum unbiased RMSE bias over Iberia under clear-sky conditions, computed for different simulations a) ERA5; b) CHTESSEL offline (CTR); c) SURFEX offline (SFX); d) H_CCl; e) H_CCI_cl; and f) $\mathrm{H}_{-} \mathrm{CCl}$ Cl_LAI. LSA-SAF LST was considered as reference for computing the simulation errors. 

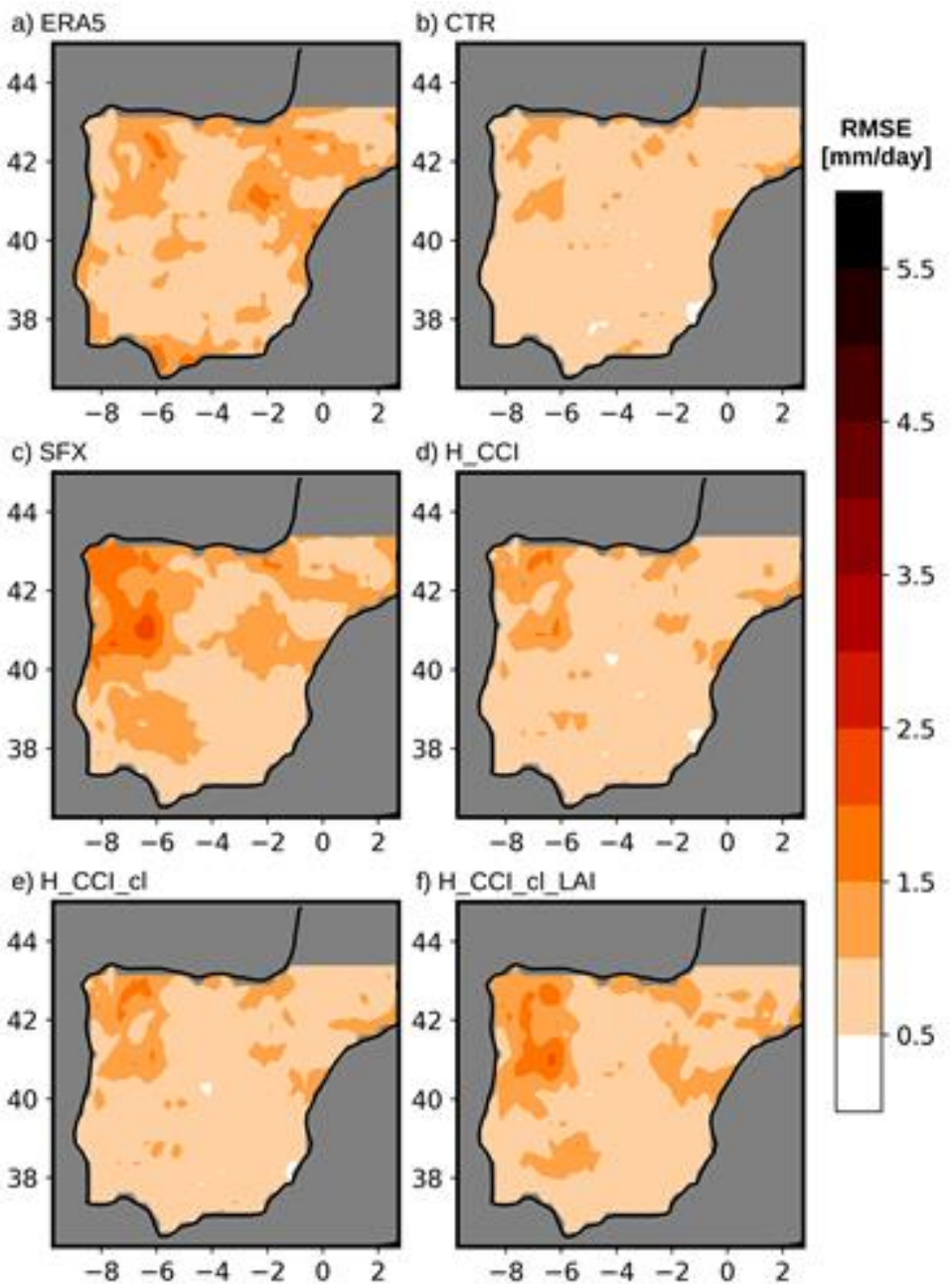

Supplementary Figure S3. JJA surface evaporation RMSE computed from a) ERA5; b) CTR; c) SFX; d) H_CCI; e) H_CCI_cl; and g) H_CCI_cl_LAI. Here GLEAMv3b dataset was considered as reference 
Supplementary Table S1. CHTESSEL land cover types and associated parameters. H/L differentiates low (L) from high $(\mathrm{H})$ vegetation; cveg is the vegetation density $(0-1)$ used in the tile fraction definition; and $\mathrm{z} 0 \mathrm{~m}$ and $\mathrm{z} 0 \mathrm{~h}$ are the roughness lengths for momentum and heat, respectively used in the calculations of the turbulent exchange coefficients for momentum, heat and water (adapted from JO19).

\begin{tabular}{|c|c|c|c|c|c|}
\hline Index & Land Cover Type & $\mathrm{H} / \mathrm{L}$ & Cveg & $\mathbf{z 0 m}$ & $\mathbf{z 0 h}$ \\
\hline 1 & Crops, mixed farming & $\mathrm{L}$ & 0.90 & 0.25 & $0.25 \times 10-2$ \\
\hline 2 & Short grass & $\mathrm{L}$ & 0.85 & 0.20 & $0.20 \times 10-2$ \\
\hline 3 & Evergreen needleleaf trees & $\mathrm{H}$ & 0.90 & 2.00 & 2.00 \\
\hline 4 & Deciduous needleleaf trees & $\mathrm{H}$ & 0.90 & 2.00 & 2.00 \\
\hline 5 & Deciduous broadleaf trees & $\mathrm{H}$ & 0.90 & 2.00 & 2.00 \\
\hline 6 & Evergreen broadleaf trees & $\mathrm{H}$ & 0.99 & 2.00 & 2.00 \\
\hline 7 & Tall grass & $\mathrm{L}$ & 0.70 & 0.47 & $0.47 \times 10-2$ \\
\hline 8 & Desert & - & 0 & 0.013 & $0.013 \times 10-2$ \\
\hline 9 & Tundra & $\mathrm{L}$ & 0.50 & 0.034 & $0.034 \times 10-2$ \\
\hline 10 & Irrigated crops & $\mathrm{L}$ & 0.90 & 0.50 & $0.50 \times 10-2$ \\
\hline 11 & Semidesert & $\mathrm{L}$ & 0.1 & 0.17 & $0.17 \times 10-2$ \\
\hline 12 & Ice caps and glaciers & - & - & $1.3 \times 10-3$ & $1.3 \times 10-4$ \\
\hline 13 & Bogs and marshes & $\mathrm{L}$ & 0.6 & 0.83 & $0.83 \times 10-2$ \\
\hline 14 & Inland water & - & - & - & - \\
\hline 15 & Ocean & - & - & - & - \\
\hline 16 & Evergreen shrubs & $\mathrm{L}$ & 0.50 & 0.10 & $0.10 \times 10-2$ \\
\hline 17 & Deciduous shrubs & $\mathrm{L}$ & 0.50 & 0.25 & $0.25 \times 10-2$ \\
\hline 18 & Mixed forest & $\mathrm{H}$ & 0.90 & 2.00 & 2.00 \\
\hline 19 & Interrupted forest & $\mathrm{H}$ & 0.90 & 1.1 & 1.1 \\
\hline 20 & Water and land mixtures & $\mathrm{L}$ & 0.60 & - & - \\
\hline
\end{tabular}

